\title{
AVALIAÇÃO DA CONCORDÂNCIA ENTRE RESSONÂNCIA MAGNÉTICA E ULTRA-SONOGRAFIA NA CLASSIFICAÇÃO DA FIBROSE PERIPORTAL EM ESOUISTOSSOMÓTICOS, SEGUNDO A CLASSIFICAC̣̃̃O DE NIAMEY*
}

\author{
Eduardo Scortegagna Junior ${ }^{1}$, Alberto Ribeiro de Souza Leão ${ }^{2}$, José Eduardo Mourão Santos ${ }^{3}$, \\ Danilo Moulin Sales ${ }^{1}$, David Carlos Shigueoka ${ }^{4}$, Luciane Aparecida Kopke de Aguiar ${ }^{5}$, Paulo \\ Eugênio Brant ${ }^{5}$, Ramiro Colleoni Neto ${ }^{6}$, Durval Rosa Borges ${ }^{7}$, Giuseppe D'Ippolito ${ }^{8}$
}

Resumo OBJETIVO: Avaliar a reprodutibilidade da ressonância magnética e a concordância entre a ultra-sonografia e a ressonância magnética na classificação da fibrose periportal em pacientes esquistossomóticos, segundo os critérios qualitativos de Niamey. MATERIAIS E MÉTODOS: Foi realizado estudo prospectivo e duplo-cego, entre fevereiro de 2005 e junho de 2006, em 20 pacientes (10 homens e 10 mulheres, idades entre 24 e 60 anos, média de 42,75 anos) com diagnóstico de esquistossomose mansônica. As imagens de ultra-sonografia e de ressonância magnética foram avaliadas por dois examinadores experientes de forma independente. Foi medida a concordância interobservador para a ressonância magnética e entre a ressonância magnética e a ultra-sonografia. RESULTADOS: A ressonância magnética apresentou resultados concordantes entre os observadores em 14 pacientes (70\%). Quando comparamos a ressonância magnética com a ultra-sonografia, obtivemos concordância em apenas seis pacientes pelo observador 1 (30\%) e em oito pacientes pelo observador 2 (40\%). CONCLUSÃO: A ressonância magnética tem boa reprodutibilidade na avaliação de fibrose periportal em pacientes com esquistossomose avançada, porém sua concordância com a ultra-sonografia é fraca.

Unitermos: Esquistossomose; Fibrose periportal; Ultra-sonografia; Ressonância magnética.

Abstract Agreement between magnetic resonance imaging and ultrasonography in the classification of schistosomal periportal fibrosis, according to Niamey's criteria.

OBJECTIVE: To evaluate the reproducibility of magnetic resonance imaging and the agreement between ultrasound and magnetic resonance imaging in the classification of periportal fibrosis in patients with schistosomiasis based on Niamey's qualitative criteria. MATERIALS AND METHODS: A prospective, double-blinded study was conducted between February 2005 and June 2006 with 20 patients (10 men and 10 women, with ages ranging between 24 and 60 years, mean age 42.7 years) diagnosed with schistosomiasis mansoni. Both ultrasound and magnetic resonance images were independently evaluated by two experienced observers. Interobserver agreement was evaluated for findings of periportal fibrosis on magnetic resonance images and in a comparison between magnetic resonance and ultrasound images. RESULTS: The analysis of magnetic resonance images showed total interobserver agreement in 14 patients $(70 \%)$. The comparison between ultrasound and magnetic resonance imaging showed agreement between images in only six cases $(30 \%)$ by observer 1 , and in eight cases $(40 \%)$ by observer 2 . CONCLUSION: Magnetic resonance imaging presents a good reproducibility in the evaluation of periportal fibrosis in later stages of schistosomiasis, however, the correlation between magnetic resonance imaging and ultrasound is poor.

Keywords: Schistosomiasis; Periportal fibrosis; Ultrasound; Magnetic resonance imaging.

\footnotetext{
* Trabalho realizado no Departamento de Diagnóstico por Imagem e nas Disciplinas de Gastroenterologia Clínica e Cirúrgica da Universidade Federal de São Paulo/Escola Paulista de Medicina (Unifesp/EPM), São Paulo, SP, Brasil.

1. Médicos Especializandos em Radiologia do Departamento de Diagnóstico por Imagem da Universidade Federal de São Paulo/Escola Paulista de Medicina (Unifesp/EPM), São Paulo, SP, Brasil.

2. Médico Pós-graduando do Departamento de Diagnóstico por Imagem da Universidade Federal de São Paulo/Escola Paulista de Medicina (Unifesp/EPM), São Paulo, SP, Brasil.

3. Mestre em Radiologia Clínica pela Universidade Federal de São Paulo/Escola Paulista de Medicina (Unifesp/EPM), São Paulo, SP, Brasil.

4. Doutor em Radiologia Clínica, Médico do Departamento de Diagnóstico por Imagem da Universidade Federal de São Paulo/Escola Paulista de Medicina (Unifesp/EPM), São Paulo, SP Brasil.
}

5. Médicos Pós-graduandos do Departamento de Gastroenterologia da Universidade Federal de São Paulo/Escola Paulista de Medicina (Unifesp/EPM), São Paulo, SP, Brasil.

6. Professor Afiliado da Disciplina de Gastroenterologia Cirúrgica da Universidade Federal de São Paulo/Escola Paulista de Medicina (Unifesp/EPM), São Paulo, SP, Brasil.

7. Professor Titular da Disciplina de Gastroenterologia, Departamento de Medicina, da Universidade Federal de São Paulo/Escola Paulista de Medicina (Unifesp/EPM), São Paulo, SP, Brasil. 8. Professor Adjunto Doutor do Departamento de Diagnóstico por Imagem da Universidade Federal de São Paulo/Escola Paulista de Medicina (Unifesp/EPM), São Paulo, SP, Brasil.

Endereço para correspondência: Prof. Dr. Giuseppe D'Ippolito. Rua Professor Filadelfo Azevedo, 617, ap. 61, Vila Nova Conceição. São Paulo, SP, Brasil, 04508-011. E-mail: giuseppe dr@ uol.com.br

Recebido para publicação em 15/12/2006. Aceito, após revisão, em 8/2/2007.

\section{INTRODUÇÃO}

A esquistossomose é uma doença milenar que afeta mais de 200 milhões de pessoas em cerca de 76 países, distribuídos pela África, Ásia e América, representando importante problema de saúde pública no Brasil $^{(1,2)}$.

A fibrose periportal é a principal causa das complicações decorrentes da esquistossomose e ocorre em $4 \%$ a $8 \%$ dos pacientes que desenvolvem infecção crônica. A 
hepatomegalia reflete a presença de inflamação granulomatosa e ocorre no início do quadro. Os depósitos de colágeno periportais levam à obstrução progressiva do fluxo sanguíneo e hipertensão portal, podendo evoluir com varizes, hemorragia digestiva alta, esplenomegalia e hiperesplenismo. A fibrose periportal pode ser identificada por meio de diversos métodos diagnósticos e é característica da doença ${ }^{(2)}$.

A ultra-sonografia (US) tem sido o exame mais utilizado nesses pacientes, revelando um padrão característico de anormalidades $^{(3)}$, sendo os achados mais importantes o espessamento ao longo das paredes da veia porta e de seus ramos, caracterizado por bandas hiperecogênicas periportais ${ }^{(4)}$.

Com o objetivo de uniformizar o diagnóstico e quantificar a fibrose periportal em pacientes esquistossomóticos, foram desenvolvidos critérios ultra-sonográficos, inicialmente no encontro de Cairo em $1990^{(\mathbf{5})}$, revistos posteriormente no encontro de Niamey em 1996. Os critérios utilizados são o qualitativo, que leva em consideração a textura hepática como um todo, e o quantitativo, que utiliza a medida objetiva da espessura das paredes de ramos da veia $\operatorname{porta}^{(\mathbf{4})}$.

Apesar de largamente adotada na avaliação de pacientes com esquistossomose, a US tem demonstrado reprodutibilidade moderada na classificação da fibrose periportal $^{(6)}$. Por outro lado, alguns trabalhos têm demonstrado a utilidade da ressonância magnética (RM) na avaliação das alterações hepatoesplênica e vasculares desse grupo de pacientes, e com elevada reprodutibilidade $^{(7)}$. Além disso, a capacidade da RM no diagnóstico da fibrose hepática tem sido bem demonstrada ${ }^{(7)}$.

No entanto, o papel da RM na caracterização e quantificação da fibrose periportal em pacientes esquistossomóticos não foi ainda estabelecido. Em virtude da sua elevada reprodutibilidade, detalhamento anatômico e elevada resolução espacial, alguns autores acreditam que a RM possa ser um método mais sensível que a US na indicação da progressão da doença, graduação e resposta à terapêutica, beneficiandose de uma avaliação mais abrangente da cavidade abdominal $^{(\mathbf{8})}$. Neste sentido, é importante estabelecer o valor da RM na avaliação da fibrose periportal.
O objetivo deste estudo foi avaliar a reprodutibilidade da RM e a concordância entre a US e a RM na classificação da fibrose periportal em pacientes esquistossomóticos, segundo os critérios qualitativos de Niamey para classificação de fibrose.

\section{MATERIAIS E MÉTODOS}

Foi realizado estudo prospectivo, transversal, observacional e duplo-cego, entre fevereiro de 2005 e junho de 2006, em 20 pacientes (10 homens e 10 mulheres, idades entre 24 e 60 anos, média de 42,75 anos) encaminhados do ambulatório de esquistossomose da Disciplina de Gastroenterologia Clínica da Universidade Federal de São Paulo/Escola Paulista de Medicina (Unifesp/EPM) e com diagnóstico de esquistossomose mansônica. O diagnóstico de esquistossomose mansônica foi obtido por meio de biópsia retal ou forte evidência clínico-laboratorial (sinais de hipertensão portal e/ou exame protoparasitológico das fezes positivo) e epidemiológica (contato com água de rios e lagoas em área endêmica) e subclassificado na forma hepatoesplênica pelo achado de hipertensão portal e esplenomegalia.

Foram excluídos os pacientes que tinham: a) contra-indicação ao exame de RM (marca-passo cardíaco, implante coclear, claustrofobia, clipe de aneurisma cerebral, alergia ao contraste paramagnético); b) antecedente de etilismo (ingestão maior que $160 \mathrm{~g}$ de etanol por semana); c) sorologia positiva para vírus $\mathrm{B}$ ou $\mathrm{C}$; d) antecedente de doença auto-imune comprovada; e) uso conhecido de droga hepatotóxica.

Os pacientes realizaram exame de US e RM com intervalo máximo de sete dias entre estes estudos. A interpretação dos resultados foi feita por dois examinadores inde- pendentes, com residência médica em diagnóstico por imagem (A.R.S.L. e D.M.S.) e experiência de pelo menos dois anos nos métodos diagnósticos empregados, com treinamento ultra-sonográfico específico para a realização do protocolo em pacientes esquistossomóticos. Num segundo momento, os examinadores se reuniram e em comum acordo atingiram consenso em relação à classificação ultra-sonográfica da fibrose periportal, o que posteriormente foi utilizado como padrão-ouro para a comparação das leituras com a RM.

\section{Exame de ultra-sonografia}

Os exames de US foram realizados com equipamento modelo EnVisor ${ }^{\circledR}$, marca Philips, utilizando-se transdutor convexo, multifreqüencial com freqüência variando entre 2,5 $\mathrm{MHz}$ e 4,5 MHz.

$\mathrm{O}$ exame foi realizado em pacientes com jejum de seis a oito horas, seguindo os planos de corte padronizados pelo protocolo de Niamey ${ }^{(\mathbf{4})}$, com acessos longitudinais (paraesternal esquerdo, hemiclavicular direito, axilar anterior direito), subesternal, subcostal, oblíquo direito e oblíquo esquerdo intercostal (Figura 1). Os padrões observados foram classificados segundo protocolo específico (Quadro 1 e Figura 2).

\section{Exame de ressonância magnética}

Os exames de RM foram realizados em equipamento operando em alto campo (1,5 T), modelo Sonata, marca Siemens, utilizando-se bobina de sinergia e seqüências de RM estabelecidas para a avaliação de fibrose periportal ${ }^{(\mathbf{9})}$ (Quadro 2).

A classificação da fibrose periportal foi feita de forma semelhante à realizada por US, utilizando-se os critérios propostos no protocolo de Niamey, anteriormente citados e adaptados às imagens de RM.

Quadro 1 Padrões do parênquima hepático e escores de fibrose periportal.

\begin{tabular}{|l|l|c|}
\hline Padrão & \multicolumn{1}{|c|}{ Imagem } & Escore FP \\
\hline A & Normal. & 0 \\
B & Céu estrelado (focos ecogênicos difusos). & 1 \\
C & Ecos anelares e em haste de cachimbo. & 2 \\
D & Hiperecogenicidade junto à bifurcação portal. & 4 \\
E & Focos altamente ecogênicos estendendo-se dos vasos portais para o & 6 \\
& parênquima. & \\
F & Bandas altamente ecogênicas estendendo-se até o parênquima, re- & 8 \\
\hline
\end{tabular}

FP, fibrose periportal. Extraído de Richter et al. ${ }^{(4)}$. 


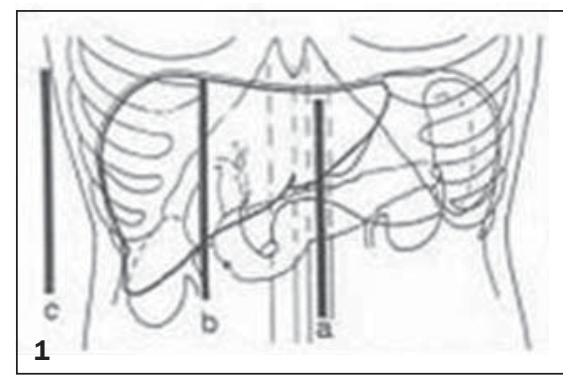

Figura 1. Planos de corte padronizados pelo protocolo de Niamey (extraído de Richter et al. ${ }^{(4)}$ ). Longitudinal: paraesternal esquerdo (1a), direito médio-clavicular (1b), axilar anterior direito (1c) transverso subesternal (2); subcostal trans-hepático (3); oblíquo direito (4); oblíquo esquerdo intercostal (5).

Os examinadores não tinham informação prévia da classificação obtida no estudo ultra-sonográfico.

A análise estatística foi feita a partir do cálculo da concordância entre os dois examinadores de RM e o padrão-ouro obtido da avaliação ultra-sonográfica em consenso.

Quadro 2 Parâmetros técnicos utilizados nas seqüências de exame.

\begin{tabular}{|l|l|}
\hline Seqüência & T2 HASTE \\
\hline TR (ms) & 800 \\
TE (ms) & 86 \\
Ângulo de inclinação ( ${ }^{\circ}$ ) & 180 \\
Número de excitações & 1 \\
2D/3D & $2 \mathrm{D}$ \\
Espessura (mm) & 7 \\
Espaçamento & 0,7 \\
Campo de visão & 350 \\
Número de cortes & $32-\uparrow$ \\
Orientação & Axial e coronal \\
Tempo de aquisição (s) & $26 / 27$ \\
Fase/freqüência & $195 / 256$ \\
Campo de visão retangular & Não \\
Saturação de gordura & Não \\
Pré-saturação & Não \\
Fourier parcial & Sim \\
Bobina & Dorso em \\
Número de medidas/repetições & $2 / 1$ \\
Circuito de disparo por ECG & Não \\
Compensação respiratória & Não \\
Ordem (cobertura do espaço K) & Half-Fourier \\
Disparo & Múltiplo \\
Comprimento do trem de eco & $195 / 256$ \\
Largura de banda (Hz/pixel) & 391 \\
Apnéia & Sim \\
\hline
\end{tabular}
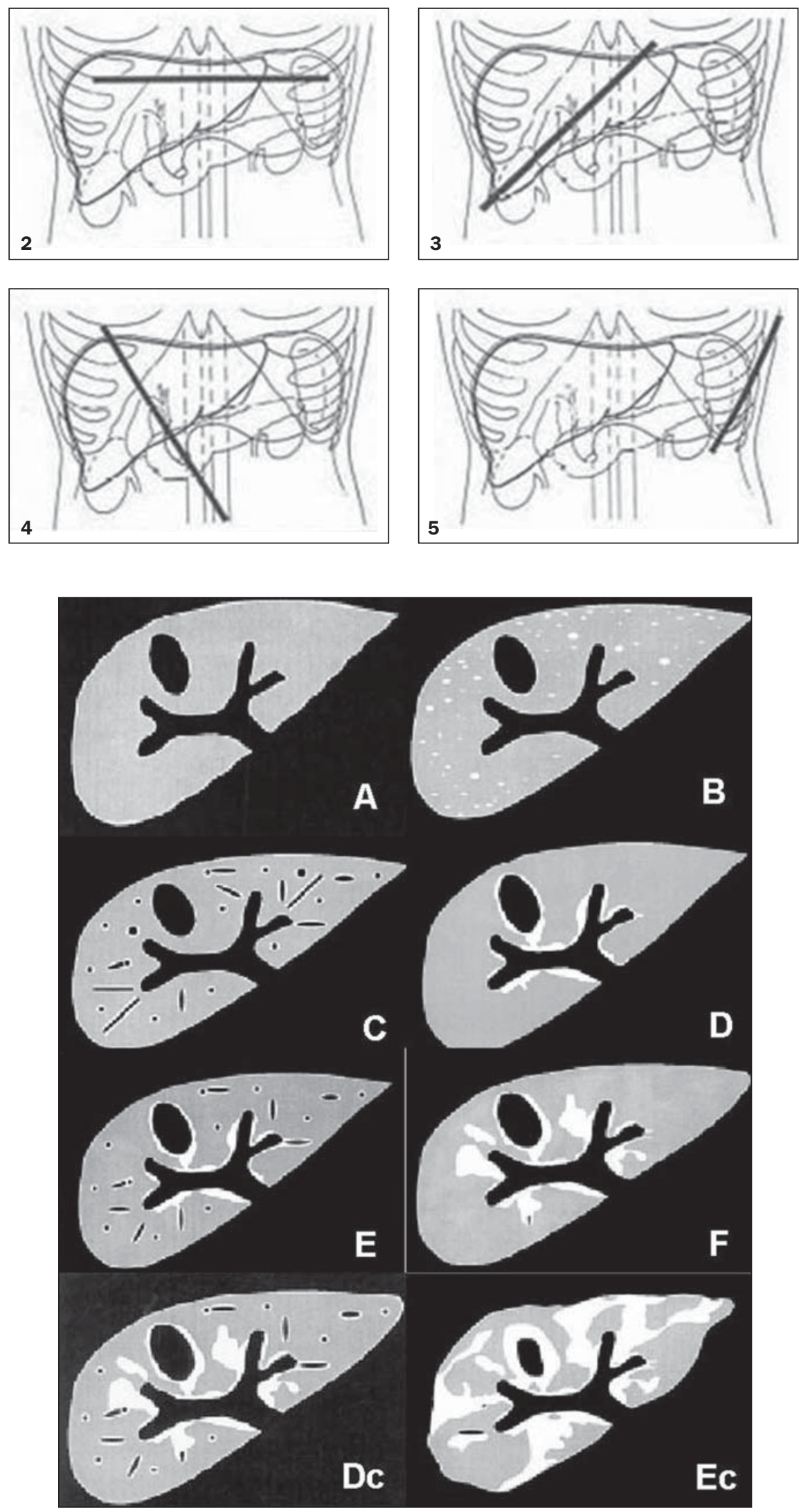

Figura 2. Normal (A); "céu estrelado" (B); anéis e "haste de cachimbo" (C); junto à bifurcação portal (D); focal (E); "garra de pássaro" (F): combinação entre $\mathbf{D}$ e $\mathbf{C}(\mathbf{D c})$; combinação entre $\mathbf{E}$ e $\mathbf{C}(\mathbf{E c})$. (Extraído de Richter et al. ${ }^{(4)}$ ). 


\section{RESULTADOS}

$\mathrm{Na}$ análise dos resultados dos exames de RM avaliados pelos leitores 1 e 2, obtevese concordância global de 70\% (14 em 20 pacientes) (Tabela 1).

Quando comparamos os resultados da RM obtidos pelo leitor 1 com o resultado ultra-sonográfico, considerado padrãoouro, obtivemos concordância fraca. Em apenas seis casos (30\%) houve concordância (Figura 3), tendo havido discrepância na classificação dos 14 pacientes restantes (70\%) (Tabela 2).

Os resultados do examinador 2 não foram muito diferentes, tendo havido concordância em apenas oito pacientes (40\%) (Tabela 3).

A maior variação de nível de classificação ocorreu em um paciente categorizado como nível $\mathrm{E}$ à US e com níveis $\mathrm{A}$ e $\mathrm{C}$ na RM (Figura 4).

As outras diferenças ocorreram de um nível de classificação para o seu subseqüente, sendo de D para E em dois casos e de E para F em três casos (Figura 5).

\section{DISCUSSÃO}

A esquistossomose é doença de grande importância nacional e internacional, pela sua elevada prevalência e pela morbidade que provoca nos pacientes em seus graus mais avançados ${ }^{(2,10)}$.

O seu tratamento geralmente leva a uma redução dos níveis de infecção e melhora em alguns achados, como a hepatoesplenomegalia e a fibrose periportal. A redução da fibrose periportal possivelmente previne o aparecimento da hipertensão portal e pode até resultar em redução da pressão portal $^{(11)}$, fato que destaca a importância da obtenção de métodos com elevada confiabilidade e reprodutibilidade para o seguimento da doença.

AUS tem sido largamente utilizada para a avaliação da doença, tendo como aliados a sua ampla disponibilidade, o baixo custo e a não-invasividade, sendo método ideal para avaliação em grandes grupos populacionais.

Este método pode ser usado para detectar a fibrose e a hipertensão portal (dilatação das veias porta e esplênica e colaterais portossistêmicas). Está provado que é mais
Tabela 1 Concordância na avaliação dos exames de ressonância magnética entre os leitores 1 e 2.

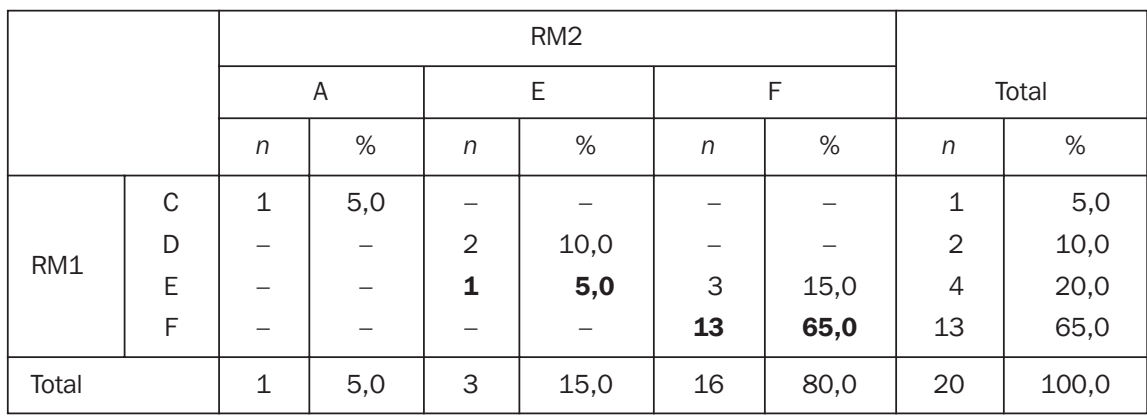

RM, ressonância magnética; $n$, número de pacientes; A-F, graus de fibrose segundo a classificação qualitativa de Niamey ${ }^{(5)}$. Obs.: Casos concordantes destacados em negrito.

Tabela 2 Resultados do leitor 1 da ressonância magnética.

\begin{tabular}{|c|c|c|c|c|c|c|c|}
\hline & & \multicolumn{4}{|c|}{ US } & & \\
\hline & & \multicolumn{2}{|c|}{$E$} & \multicolumn{2}{|c|}{$\mathrm{F}$} & \multicolumn{2}{|c|}{ Total } \\
\hline & & $n$ & $\%$ & $n$ & $\%$ & $n$ & $\%$ \\
\hline \multirow{4}{*}{ RM } & C & 1 & 5,0 & 0 & 0,0 & 1 & 5,0 \\
\hline & D & 1 & 5,0 & 1 & 5,0 & 2 & 10,0 \\
\hline & $E$ & 1 & 5,0 & 3 & 15,0 & 4 & 20,0 \\
\hline & $\mathrm{F}$ & 8 & 40,0 & 5 & 25,0 & 13 & 65,0 \\
\hline \multicolumn{2}{|c|}{ Total } & 11 & 55,0 & 9 & 45,0 & 20 & 100,0 \\
\hline
\end{tabular}

US, ultra-sonografia; RM, ressonância magnética; $n$, número de pacientes; A-F, graus de fibrose segundo a classificação qualitativa de Niamey ${ }^{(5)}$. Obs.: Casos concordantes destacados em negrito.

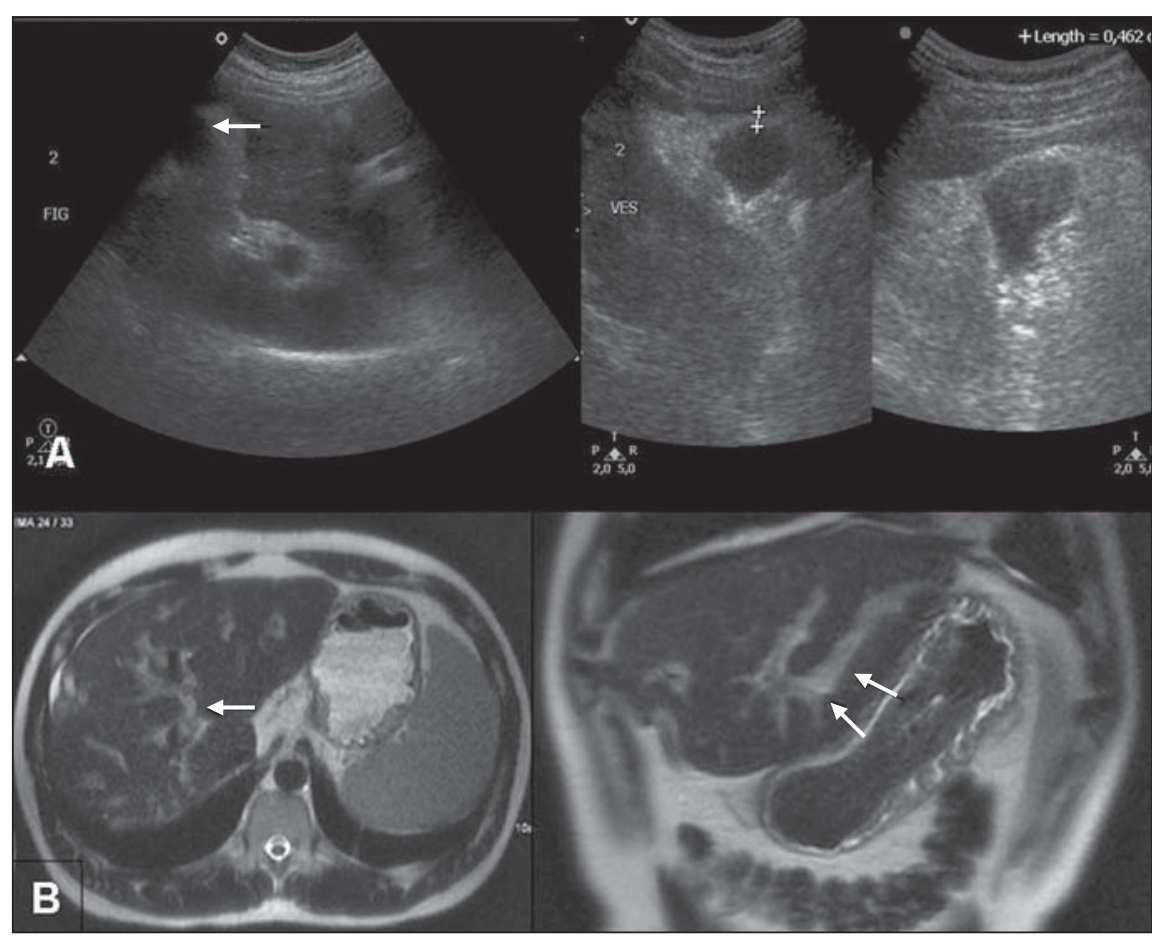

Figura 3. Ultra-sonografia $(\mathbf{A})$ e ressonância magnética $(\mathbf{B})$ de paciente com classificação $F$ aos dois métodos. Aspecto da fibrose periportal (setas). 
Tabela 3 Resultados do leitor 2 da ressonância magnética.

\begin{tabular}{|c|c|c|c|c|c|c|c|}
\hline & \multicolumn{4}{|c|}{ US } & & \\
\hline & & \multicolumn{2}{|c|}{ E } & \multicolumn{2}{|c|}{$\mathrm{F}$} & \multicolumn{2}{|c|}{ Total } \\
\hline & & $n$ & $\%$ & $n$ & $\%$ & $n$ & $\%$ \\
\hline \multirow{3}{*}{ RM } & A & 1 & 5,0 & 0 & 0,0 & 1 & 5,0 \\
\hline & $E$ & 1 & 5,0 & 2 & 10,0 & 3 & 15,0 \\
\hline & $\mathrm{F}$ & 9 & 45,0 & 7 & 35,0 & 16 & 80,0 \\
\hline \multicolumn{2}{|c|}{ Total } & 11 & 55,0 & 9 & 45,0 & 20 & 100,0 \\
\hline
\end{tabular}

US, ultra-sonografia; RM, ressonância magnética; $n$, número de pacientes; A, E, F, graus de fibrose segundo a classificação qualitativa de Niamey ${ }^{(5)}$. Obs.: Casos concordantes destacados em negrito.

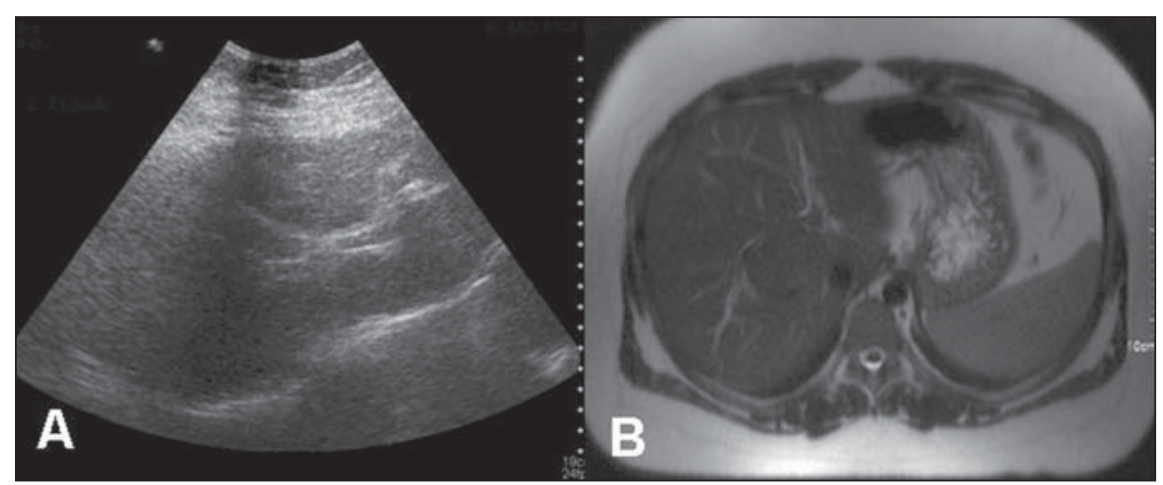

Figura 4. Ultra-sonografia (A) e ressonância magnética (B) de paciente com classificação ultra-sonográfica E e leituras discordantes de ressonância magnética entre os examinadores (respectivamente classificados como C e A).

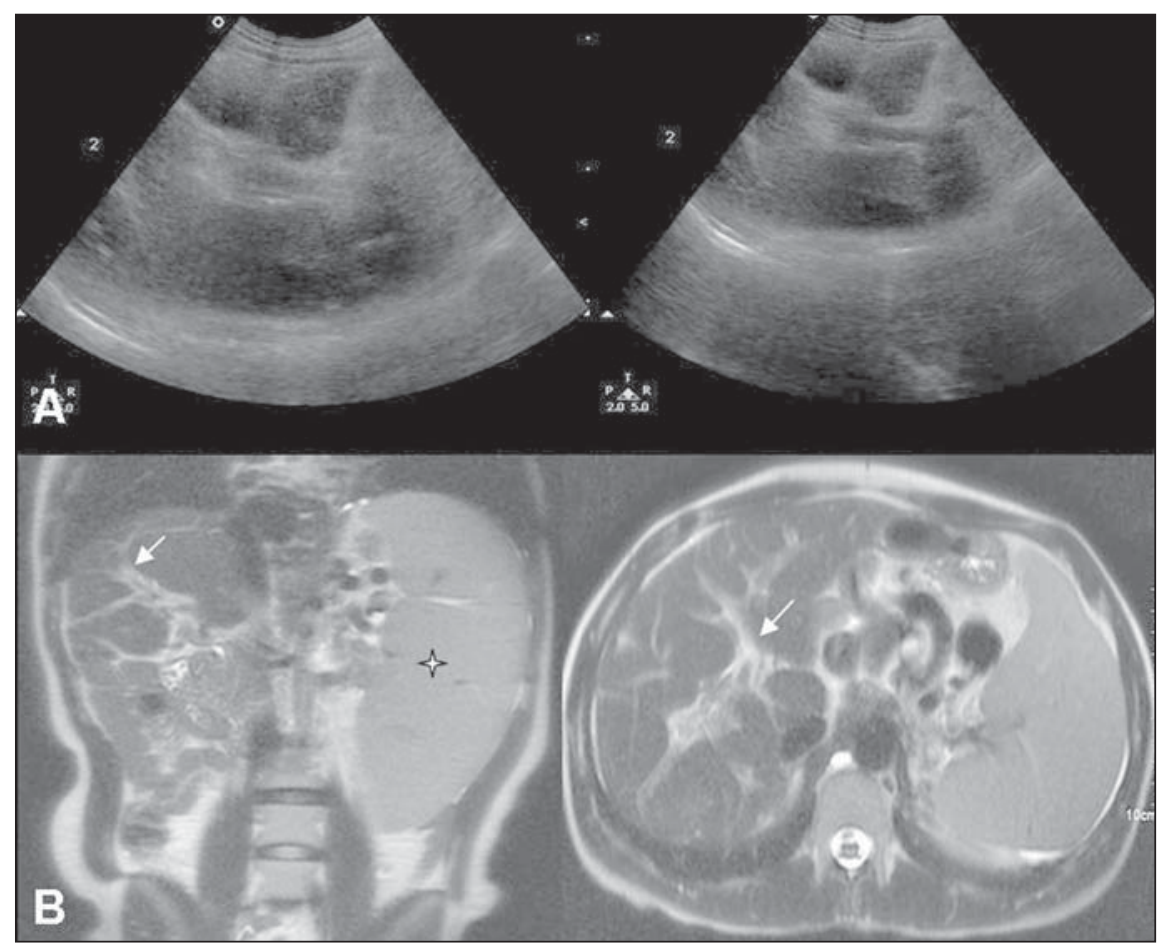

Figura 5. Ultra-sonografia (A) e ressonância magnética (B) de paciente com graduação $E$ à ultra-sonografia e $\mathrm{F}$ à ressonância magnética, tendo havido concordância interobservador na ressonância magnética. Aspecto da fibrose periportal (setas). Notar a acentuada esplenomegalia (estrela). confiável do que métodos clínicos para o diagnóstico de doenças hepatoesplênicas. A fibrose periportal, que é a lesão essencial observada na esquistossomose, é geralmente identificada anos após a infecção, porém já tendo sido relatada em fases mais precoces da infestação ${ }^{(4)}$. Por outro lado, a US tem demonstrado reprodutibilidade apenas moderada na avaliação de pacientes com esquistossomose ${ }^{(\mathbf{6})}$.

Já a RM tem, progressivamente, se firmado como método extremamente útil na avaliação das hepatopatias focais ou difusas do fígado ${ }^{(\mathbf{1 2})}$, e com elevada reprodutibilidade $^{(\mathbf{1 3})}$, necessária para se obter resultados consistentes e estimular a adoção do método. De maneira semelhante, a RM tem mostrado ser capaz de identificar com bastante precisão a fibrose hepática ${ }^{(7,12)}$. Apesar disso, não existem, até o momento, trabalhos na literatura que tenham comparado a capacidade da RM e da US na avaliação da fibrose periportal, sendo este o primeiro do qual temos conhecimento.

Os aspectos da fibrose portal já foram descritos. Nas seqüências ponderadas em T1 a fibrose se apresenta como uma banda hipointensa em relação ao fígado, enquanto na seqüência T2 apresenta sinal elevado ${ }^{(11)}$, sendo as imagens em T2 as mais amplamente utilizadas na literatura com este objetivo $^{(\mathbf{8 , 9 , 1 3 , 1 4})}$, fato este observado no nosso estudo, porém com fraca concordância com os achados ultra-sonográficos.

O nosso estudo apresenta algumas limitações que devem ser apontadas. Todos os pacientes tiveram classificação ultra-sonográfica $\mathrm{E}$ ou $\mathrm{F}$ de Niamey, o que caracteriza um estágio avançado de fibrose periportal, limitando a avaliação do método a esse grupo de pacientes. Não sabemos como o método se comportaria na avaliação de pacientes com graus mais brandos de fibrose periportal.

Isso também impossibilitou o cálculo do kappa para concordância interobservador da RM, pois não houve pacientes com todos os níveis de fibrose na classificação de Niamey. Por outro lado, os pacientes que mais se beneficiariam de uma avaliação abrangente, como aquela fornecida pela $\mathrm{RM}$, seriam aqueles com formas mais avançadas da doença e com maior risco de desenvolver complicações e que foi justamente o grupo estudado. 
A adaptação dos critérios ultra-sonográficos de Niamey para serem utilizados na $\mathrm{RM}$ revelou boa reprodutibilidade do método $(70 \%)$, não se tendo observando, no entanto, relação satisfatória entre a classificação obtida pela RM e pela US. Houve concordância entre os dois métodos em menos da metade dos casos estudados. Uma possível explicação para esse resultado pode ser encontrada observando-se a tênue variação morfológica entre as classificações E e F, cuja única diferença é a retração do parênquima em $\mathrm{F}$, achado esse, assim como os demais, de certa subjetividade. É importante observar que, mesmo utilizando-se a US, observa-se uma variabilidade considerável entre observadores, o que foi demonstrado em outro estudo desenvolvido pelo nosso grupo ${ }^{(6)}$. Outro fator a ser considerado é que partimos do pressuposto que a US é o método padrão de referência para avaliar fibrose periportal, apesar de não haver consenso neste sentido. Será necessário estabelecer a correlação entre a US, a RM e a análise anatomopatológica da fibrose periportal para esclarecer qual método apresenta realmente a maior eficácia.

Acreditamos também que ulteriores estudos deverão ser desenvolvidos no sentido de se estabelecer uma proposta de modelo de classificação simplificado para fibrose periportal, e que atenda as necessidades dos profissionais que acompanham este grupo de pacientes, assim como trabalhos com um maior número de pacientes com doença em fase inicial, para se validar a eventual utilidade da RM.

Concluindo, na avaliação da fibrose periportal, através de critérios de Niamey adaptados para RM, o método apresentou boa reprodutibilidade, porém, baixa concordância com a US, o que torna a sua aplicabilidade ainda discutível, principalmente em pacientes com esquistossomose.

\section{REFERENCIAS}

1. Alves Jr A, Fontes DA, Melo VA, Machado MCC, Cruz JF, Santos EAS. Hipertensão portal esquistossomótica: influência do fluxo sanguiíneo portal nos níveis séricos das enzimas hepáticas. Arq Gastroenterol 2003;40:203-208.

2. Ross AG, Bartley PB, Sleigh AC, et al. Schistosomiasis. N Engl J Med 2002;346:1212-1220.

3. Homeida M, Ahmed S, Dafalla A, et al. Morbidity associated with Schistosoma mansoni infection as determined by ultrasound: a study in Gezira, Sudan. Am J Trop Med Hyg 1988;39: 196201.

4. Richter J, Hatz C, Campagne G, Berquist NR, Jenkins JM. Ultrasound in schistosomiasis. A practical guide to the standardized use of ultrasonography for the assessment of schistosomiasis-related morbidity. Second International Workshop, Niamey, Niger, October 22-26, 1996.

5. International conference on schistosomiasis. Cairo, Egito, 1995.
6. Santos GT, Sales DM, Shigueoka DC, et al. Reprodutibilidade da classificação ultra-sonográfica de Niamey na avaliação da fibrose periportal na esquistossomose mansônica. Radiol Bras 2006;39 (Supl 2):57.

7. Bezerra ASA, D'Ippolito G, Caldana RP, Cecin AO, Szejnfeld J. Avaliação hepática e esplênica por ressonância magnética em pacientes portadores de esquistossomose mansônica crônica. Radiol Bras 2004;37:313-321.

8. Patel SA, Castillo DF, Hibbeln JF, Watkins JL. Magnetic resonance imaging appearance of hepatic schistosomiasis, with ultrasound and computed tomography correlation. Am J Gastroenterol 1993;88:113-116.

9. Kashitani N, Kimoto S, Tsunoda M, et al. Portal blood flow in the presence or absence of diffuse liver disease: measurement by phase contrast MR imaging. Abdom Imaging 1995;20:197-200.

10. Hatz CF. The use of ultrasound in schistosomiasis. Adv Parasitol 2001;48:225-284.

11. Homeida MMA, Eltoum IA, Ali MM, et al. The effectiveness of annual versus biennial mass chemotherapy in reducing morbidity due to schistosomiasis: a prospective study in Gezira-Managil, Sudan. Am J Trop Med Hyg 1996;54:140-145.

12. Vitellas KM, Tzalonikou MT, Bennett WF, Vaswani KK, Bova JG. Cirrhosis: spectrum of findings on unenhanced and dynamic gadolinium-enhanced MR imaging. Abdom Imaging 2001;26: 601-615.

13. Lambertucci JR, Silva LC, de Queiroz LC, PintoSilva RA. Magnetic resonance imaging and ultrasound in hepatosplenic schistosomiasis mansoni. Rev Soc Bras Med Trop 2004;37:333-337.

14. Herborn CU, Akkoyunlu B, Ruehm G. Schistosoma mansoni-Befall der Leber - Darstellung mittels Magnetresonanztomographie (MRT). Rofo 2002;174:490-494. 